Results The distribution of stented vessels, diameter of reference vessels, and post-procedural minimal lumen diameter were compared in each group. At week 4 follow-up, quantify coranary angiography (OCA) showed that the minimal lumen diameter were greater, and percent stenosis and late lumen loss in the triptolidecoated stent group and DES group were less than that of the bare metal stents group. In the histopathologic analysis, compared to BMS group, the injury score, inflammation score in TCS group and DES group were decreased, $\mathrm{p}<0.05$, and there were significant reduced neointimal area and enlarged Lumen area in TCS, DES group as well.

Conclusion Triptolide-coated stent showed the similar effect of preventing restenosis through inhibition of neointimal hyperplasia as DES and anti-inflammation acts after coronary angioplasty in pigs.

\section{e0510 RELATION BETWEEN CLOPIDOGREL RESISTANCE AND INFLAMMATION FACOR IN PATIENTS AFTER CORONARY STENTING}

doi:10.1136/hrt.2010.208967.510

Ge Hailong, Zhou Yujie, Zhao Yinxin, Shi Dongmei, Liu Yuyang, Guo Yonghe, Yang Oing, Cheng Wanjun. Department of Cardiology, Anzhen Hospital, Capital Medical University, China

Objective To observe the serum levels of inflammatory cytokines in patients with clopidogrel resistance underwent PCI.

Methods Platelet aggregation (PAG) induced with ADP were detected in 593 patients with unstable angina pectoris and had received coronary stenting. All patients were divided into clopidogrel resistance (CLR) group $(n=333)$ and normal response group $(n=260)$ according to PAG. At the same time, perpheral blood sample of all the patients were taken before, $24 \mathrm{~h}$, 1 week and 1 month after implanting stents. The levels of C-reactive protein (CRP), P-selection and soluble fragment of CD40 ligand (sCD40L) in these patients were detected by enzyme linked immunosorbent assay.

Results The incidence of clopidogrel resistance in $24 \mathrm{~h}, 1$ week and 1 month after PCI was $35.5 \%, 26.9 \%$ and $19.3 \%$. The levels of CRP $(8.8 \pm 2.5 \mathrm{mg} / \mathrm{dl}$ at $24 \mathrm{~h}, 5.3 \pm 2.5 \mathrm{mg} / \mathrm{dl}$ at 1 week), P-selection $(73.8 \pm 34 \mathrm{ng} / \mathrm{ml}$ at $24 \mathrm{~h}, 70.5 \pm 31.6 \mathrm{ng} / \mathrm{ml}$ at 1 week and $66.4 \pm 22.3 \mathrm{ng} / \mathrm{ml}$ at 1 month) and sCD $40 \mathrm{~L}(7.7 \pm 2.3 \mathrm{ng} / \mathrm{ml}$ at $24 \mathrm{~h})$ had significant difference compared with the levels before and normal group $(\mathrm{p}<0.05)$. The CLR at 30 days after stent implanting was significantly correlated with the level of P-selection $(r=1.334)$ and smoking ( $\mathrm{r}=1.053)$.

Conclusion The levels of CRP, P-selection and sCD40L of in some patient after undergoing coronary stenting increased and may correlated with clopidogrel resistance. The levels of P-selection and smoking were the predictor for clopidogrel resistance.

\section{e0511 IMPACT OF SMOKING ON PLATELET INHIBITION OF CLOPIDOGREL IN PATIENTS UNDERGOING PERCUTANEOUS CORONARY INTERVENTION}

doi:10.1136/hrt.2010.208967.511

Liu Xiaoli, Zhou Yujie, Yang Qing, Yang Shiwei, Jia Dean. Department of Cardiology, Beijing Anzhen Hospital, Capital Medical University, Beijing, China

Objective The study aimed to evaluate the impact of smoking on platelet inhibition of clopidogrel.

Methods The clopidogrel-native patients were enrolled. Cigarette smoking status was recorded and stratified into never smoking (NS) and current smoking (CS). All the patients were given loading dose of $300 \mathrm{mg}$ and maintenance dose of $75 \mathrm{mg}$ clopidogrel. Platelet aggregation was measured before and $24 \mathrm{~h}$ after loading by conventional light transmittance aggregometry after stimulation with $5 \mathrm{mmol} / \mathrm{L}$ ADP.

Results 722 patients (60.7 \pm 9.0 years) were enrolled, including 568 men $(78.7 \%)$. Among the patients, 322 patients (44.6\%) were CS and $400(55.4 \%)$ were NS. At baseline, the maximal platelet aggregation (MPA) was not significantly different between CS and NS (57.2 $\pm 10.4 \%$ and $56.6 \pm 12.8 \%, \mathrm{p}=0.487$ ). After clopidogrel loading, the MPA decreased significantly among CS compared with NS. The mean decreased MPA was $23.8 \pm 10.5 \%$ and $19.2 \pm 12.8 \%(\mathrm{p}<0.001)$ for CS and NS, respectively.

Conclusion Cigarette smoking might enhance the inhibition effect of clopidogrel on platelet.

\section{e0512 IMPACT OF SMOKING-CESSATION ON PLATELET INHIBITION OF CLOPIDOGREL IN PATIENTS UNDERGOING ELECTIVE CORONARY DRUG-ELUTING STENT IMPLANTATION}

doi:10.1136/hrt.2010.208967.512

Liu Xiaoli, Zhou Yujie, Yang Oing, Yang Shiwei, Gao Fei. Department of Cardiology, Beijing Anzhen Hospital, Capital Medical University, Beijing, China

Objective Cigarette smoking, as an inducer of CYP1A2 and increase the CYP1A2 activity, might enhance the generation of the active metabolite of clopidogrel. The study aimed to examine the impact of smoking cessation on platelet inhibition of clopidogrel.

Method Current smoking patients undergoing elective coronary stent implantation were enrolled. Every patient was aggressively advised to quit smoking at admission. All the patients were given loading dose of $300 \mathrm{mg}$ clopidogrel and $300 \mathrm{mg}$ aspirin and daily maintenance dose of $75 \mathrm{mg}$ clopidogrel in addition to $100 \mathrm{mg}$ aspirin. Blood samples were collected before and $24 \mathrm{~h}$ after clopidogrel loading and pre-discharge. Platelet aggregation was determined by conventional light transmittance aggregometry after stimulation with $5 \mathrm{mmol} / \mathrm{L}$ ADP.

Results 224 male smoking patients were enrolled in our study. During staying in hospital, 157 patients gave up smoking and 67 still kept smoking. Before loading, the maximal platelet aggregation (MPA) was not significantly different between non-smokers and smokers $(56.4 \pm 12.3 \%$ and $58.2 \pm 13.8 \%, \mathrm{p}=0.357)$. However, MPA after clopidogrel loading $(42.8 \pm 11.5 \%$ and $38.6 \pm 14.8 \%, p=0.038)$ and pre-discharge $(40.2 \pm 10.3 \%$ and $36.5 \pm 13.2 \%, p=0.041)$ showed significant difference between two groups.

Conclusion Smoking cessation might weaken inhibition effect of clopidogrel on platelet aggregation for smokers.

\section{e0513 PLASMA NGAL COULD EARLY PREDICT CONTRAST- INDUCED ACUTE KIDNEY INJURY AFTER PERCUTANEOUS CORONARY INTERVENTIONS}

doi:10.1136/hrt.2010.208967.513

Liu Xiaoli, Zhou Yujie, Wang Zhijian, Nie Bin. Department of Cardiology, Beijing Anzhen Hospital, Capital Medical University, Beijing, China

Objective To examine the changing trend of plasma Neutrophil gelatinase-associated lipocalin (NGAL) after contrast exposure and to elucidate whether NGAL was good early biomarker for diagnosis of contrast induced acute kidney injury (CIAKI).

Method 231 patients undergoing percutaneous coronary intervention were enrolled. Blood samples were collected before and 2, 4, 8,24 , and $48 \mathrm{~h}$ after procedure. NGAL was measured with ELISA kit. CIAKI was defined as an increase in the serum creatinine values of $\geq 25 \%$ or $\geq 44.2 \mu \mathrm{mol} / \mathrm{L}$. Receiver-operating characteristic (ROC) 